\title{
Optimization of Dynamic Resource Allocation and Rate Adaptation in OFDMA Systems
}

\author{
M.Vadivel \\ Research Scholar, \\ Sathyabama University, \\ Chennai - 600119, India,
}

\author{
H.Ranganathan \\ Professor and Head, ECE Department, \\ Sakthi Mariamman Engineering College, \\ Chennai - 602 105, India,
}

\begin{abstract}
To support high data rate applications under limited radio resources and rough wireless channel conditions, dynamic resource allocation is one of the promising techniques which achieve both system capacity and QoS requirements. Apart from the resource allocation techniques, it is imagined that incorporation of rate and power adaptation mechanisms can significantly improve the system performance under fading channels and thereby make the system more robust to deep fading events. In this work it is proposed about how dynamic resource allocation can be implemented in OFDMA systems with fairly in low complexity and with a better performance in terms of the BER and quality of service to the users.
\end{abstract}

\section{Keywords}

OFDMA, QoS, BER, CSI, ISI

\section{INTRODUCTION}

In recent years, huge demands for fast and reliable communications over wireless channels. OFDMA is a method that assigns different groups of subcarriers (in frequency) to different users. This ensures that more than one user can access the air interface at the same time. In OFDM all $\mathrm{K}$ subcarriers are used to carry data for one user only. OFDM assigns all subcarriers to a single user at the same time, and only one user can transmit at a time. If multiple users want to transmit using OFDM, then those users have to take their turns in time. .In OFDMA, instead of sequentially assigning OFDM symbols in time to different users, the system directly assigns subcarriers in frequency to different users.

Dardari D [13] has suggested Ordered Subscriber Selection Algorithm (OSSA). This is a low-complexity and lowoverhead adaptive bit-loading algorithm for wireless multicarrier schemes. It is based on the diversity gain of the more reliable subcarriers. It is found that if this algorithm is used, there is wastage of bandwidth. Besides, it is found that this algorithm works well only when CSI is available in the transmitter.

Natalia Y. Ermolova and Boris Makarevitch [7] have addressed the problem of low complexity sub-optimal power and subcarrier allocation for OFDMA systems. They claim that their method improves the average BER-performance of the system besides providing fairness between the users in terms of the bit rates. This algorithm also results in wastage of bandwidth apart from the fact that the system assumes prior knowledge of CSI (channel state information) at the transmitter.

\subsection{Aim and Scope}

The goal of this project is to implement adaptive bit loading mechanisms in conventional OFDMA systems. The adaptation techniques used in past works are non trivial in implementation in terms of the computational complexity. Here a modified algorithm is presented and its output performance over the existing mechanisms is proved via link level simulations.

In earlier works, they improve the performance of the system and increase the computational complexity. Also here the idea of bit loading [1] is incorporated in OFDMA systems where the adaptive power loading is used in existing systems. The simulation results show that the proposed mechanisms provide better BER performance with low computational complexity.

\section{SYSTEM MODEL}

The variation of wireless channels used to be regarded as a fundamental limitation on the performance of wireless systems, as bit error rate (BER) is typically dominated by the worst-case channel condition. With adaptive resource allocation [2][5], however, the time and frequency-varying characteristics of wireless channels can be exploited and indeed considered an advantage.

\subsection{System Model of Multi-User OFDMA}

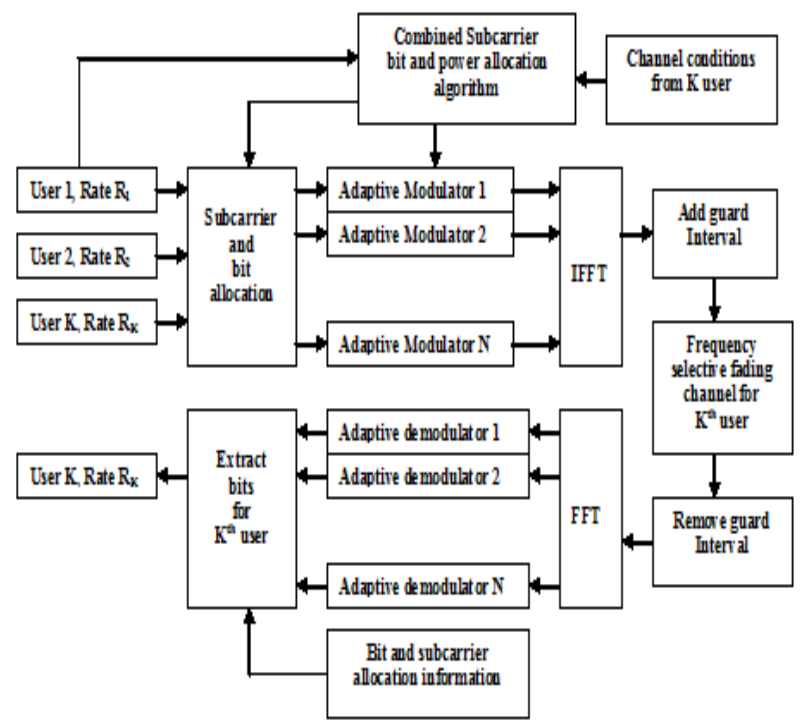

Figure 1. Block Diagram of MU-Adaptive OFDMA system

The configuration of multi-user adaptive OFDMA system is shown in Figure 1. We assume that the system has $\mathrm{K}$ users and the $\mathrm{k}^{\text {th }}$ user has a data rate $\mathrm{R}_{\mathrm{k}}$ equal to bit per OFDMA symbol. In the transmitter, the serial data from the users are fed into the subcarrier and bit allocation block which allocates bits [3] from different users to different subcarriers. 
We assume that each subcarrier has a bandwidth that is much smaller than the coherence bandwidth of the channel and that the instantaneous channel gains on all the subcarriers of all the users are known to the transmitter. Using the channel information, the transmitter applies the combined subcarrier, bit, and power allocation algorithm [4][12] to assign different subcarriers to different users and the number of bits/OFDMA symbol to be transmitted on each subcarrier.

Depending on the number of bits assigned to a subcarrier, the adaptive modulator will use a corresponding modulation scheme, and the transmit power level will be adjusted according to the combined subcarrier, bit, and power allocation algorithm [10].

We define $C_{k, n}$ to be the number of bits of the $\mathrm{k}^{\text {th }}$ user that are assigned to the $\mathrm{n}^{\text {th }}$ subcarrier. As we do not allow more than one user to share a subcarrier, it follows that for each n, if $_{\mathrm{k}, \mathrm{n} \neq 0}, \mathrm{C}_{\mathrm{k}, \mathrm{n}=0}$, for all $\mathrm{k}=\mathrm{k}$ '. We also assume that the adaptive modulator allows $C_{k, n}$ to take values in the set $D=\{0,1,2$, .M) where is the maximum number of information bits/OFDMA symbol that can be transmitted by each subcarrier. The complex symbols at the output of the modulators are transformed into the time domain samples by inverse fast Fourier transform (IFFT). Cyclic extension of the time domain samples, known as the guard interval, is then added to ensure orthogonally between the subcarriers, provided that the maximum time dispersion is less than the guard interval. The transmit signal is then passed through different frequency selective fading channels to different users.

We assume that the subcarrier and bit allocation information is sent to the receivers via a separate control channel. At the receiver, the guard interval is removed to eliminate the ISI, and the time samples of the $\mathrm{k}^{\text {th }}$ user are transformed by the FFT block into modulated symbols. The bit allocation information is used to configure the demodulators while the subcarrier allocation information is used to extract the demodulated bits from the subcarriers assigned to the $\mathrm{k}^{\text {th }}$ user.In the frequency selective fading channel [7], different subcarriers will experience different channel gains. We denote by $\mathrm{C}_{\mathrm{k}, \mathrm{n}}$ the magnitude of the channel gain (assuming coherent reception) of the nth subcarrier as seen by the $\mathrm{K}^{\text {th }}$ user.

We denote the required received power (in energy per symbol) in a subcarrier [8] for reliable reception of information bits/symbol when the channel gain is equal to unity. Note that the function depends on, and this allows different users to have different quality-of-service (QoS) requirements and/or different coding and modulation [6] schemes.

In order to maintain the required QoS at the receiver, the transmit power, allocated to the $\mathrm{n}^{\text {th }}$ subcarrier by the $\mathrm{k}^{\text {th }}$ user must equal

$$
P_{k, n}=\frac{f_{k}\left(c_{k, n}\right)}{\alpha_{k, n}^{2}}
$$

Using these transmit power levels, the receiver can demodulate the modulated symbols at the output of the FFT processor and achieve the desired QoS's of all users. The goal of the combined subcarrier, bit, and power allocation algorithm[4][12] is then to find the best assignment of $C_{k, n}$ so that the overall transmit power, the sum of overall subcarriers $\mathrm{p}_{\mathrm{k}, \mathrm{n}}$ and all users, is minimized for given transmission rates of the users and given QoS requirements specified through $f_{k}() \mathrm{k}=1, \ldots \ldots, \mathrm{k}$. In order to make the problem tractable, we further require that $f_{k}(c)$, is a convex and increasing function with $f_{k}(0)=0$.

This condition essentially means that no power is needed when no bit is transmitted and that the required additional power to transmit an additional bit increases with [i.e., is increasing in]. Almost all popular coding and modulation schemes satisfy this condition. It is important to note that even though the problem is formulated to minimize the overall transmit power for given QoS requirements, the same solution can be applied to improve the QoS's of the users for a given overall transmit power. The latter can simply be achieved by increasing the power proportionally for all the subcarriers, while using the same setoff $C_{k, n}$.

\section{DESIGN METHODOLOGIES}

\subsection{Rate Power Adaptation Mechanism}

Multicarrier modulation is the method of choice for many data transmission systems, including wireless local area networks (WLAN) applications. In conventional wireless orthogonal frequency division multiplexing systems, all subcarriers employ the same signal constellation. However, the overall error probability is dominated by the subcarriers with the worst performance. To improve performance, adaptive bit and power allocation (i.e., "loading") algorithms [4][12] can be employed, where the signal constellation size and power distribution vary according to the measured signalto-noise ratio (SNR) values across the subcarriers. In extreme situations, some subcarriers can be "turned off" or nulled if the subcarriers values are poor.

Most published proposals of bit and power allocation algorithms are variants of three basic types of algorithms: incremental(i.e., "greedy") allocation bit loading based on channel capacity approximations and bit loading based on probability of bit error expressions .The first type incrementally allocates an integer number of bits at the cost of high computational complexity. The other two types of algorithms use closed-form expressions of performance measures in order to determine a bit allocation but require rounding to integer values, which may lead to allocations that are far from the optimum.

Common choices for objective functions that loading algorithms are attempting to optimize are the maximization of the overall throughput given a total power constraint, known as rate-adaptive loading, and the minimization of the energy given a fixed throughput, known as margin-adaptive loading. In this work, we propose two discrete rate-adaptive loading algorithms that try to balance the implementation tradeoffs while coming close to the maximum throughput.

However, the introduction of coding would improve the performance relative to an uncoded system and can be accounted for by a nonlinear modification of the SNR values in relationship to the coding gain. Many adaptive allocation algorithms can perform both adaptive bit and power loading; the algorithms studied and evaluated in this paper employ only adaptive bit loading [1] and uniform power allocation.

\subsection{Adaptive bit Loading}

The possible solutions for using Adaptive Bit Loading is a. More number of bits is made to go through better part of the channel (Bit Allocation)

b. More energy is allocated to sub channels which are corrupt. (Energy Allocation) 
The reason of allocating more bits to better sub channel is that, since these channels have better SNR, large number of bits can be received with high degree of error-freeness. At the same time, by allocating more energy to poorer sub carriers, you are increasing signal strength of such sub channels so much that they can overcome noise that will be added by the channel.

\section{IMPLEMENTATION OF THE PAPER}

The implementation of loading algorithms is usually a tradeoff between how close they come to the optimum allocation and how quickly they reach their final allocation.

\subsection{Proposed Power Loading Algorithm}

The expression for BER of square M-QAM with Gray bit mapping can be approximated by the equation

$$
P_{i}=0.6 \exp \left\{-\frac{1.8 \alpha_{i}}{2^{b_{i}}-1}\right\} \text { Where } i=1,2 \ldots . . N
$$

Where $\alpha_{i}=\frac{P_{i}\left|H_{i}\right|^{2}}{\sigma_{i}^{2}}$ is the $i^{\text {th }}$ sub channel SNR. Pi, Hi and $\sigma_{i}$ are the signal power, channel gain and noise power of the $i^{\text {th }}$ subcarrier respectively. The numbers of bits that can be carried in subchannel can be calculated using the equation

$$
b_{i}=\log _{2}\left\{1+\frac{\alpha_{i}}{\Gamma_{i}}\right\} \text { Where } i=1,2 \ldots . . N
$$

Where $\Gamma_{i}$ is the SNR gap. It represents how far the system is from achieving capacity. It can be defined from the above two equations

$$
\Gamma_{i}=-\frac{\ln \left(6 . P_{i}\right)}{1.8} \text { Where } i=1,2 \ldots . . N
$$

The average number of bits per subcarrier in one OFDMA symbol can then be found as

$$
\bar{b}=\log _{2}\left\{1-\frac{1.8 \alpha_{m c}}{\ln (6 . \bar{P})}\right\}
$$

In this expression, $\alpha_{m c}$ is the multichannel SNR that characterizes the set of $N$ subchannels by an equivalent single AWGN that achieves the same data rate with the same error probability. It is given by

$$
\alpha_{\mathrm{mc}}=\frac{\ln \left(6 . \mathrm{P}_{\mathrm{i}}\right)}{1.8}\left\{1-\left[\prod_{\mathrm{i}=1}^{\mathrm{N}}\left(1-\frac{1.8 \alpha_{\mathrm{i}}}{\ln \left(6 . \mathrm{P}_{\mathrm{i}}\right)}\right)\right]^{(1 / \mathrm{N})}\right\}
$$

For each subcarrier, the proposed algorithm initially computes the maximum number of bits $b_{i}$ that gives a value of $P_{i}$ below $\mathrm{P}_{\mathrm{T}}$. It is expected that the resulting overall BER $\bar{P}_{\text {will be }}$ below $\mathrm{P}_{\mathrm{T}}$ by an appreciable margin. To make maximum use of this margin, the algorithm then computes the number of extra bits that can be added to the OFDMA symbol without violating the $B E R$ constraint $P_{T}$.

The proposed algorithm proceeds along the following steps:

1) For the given values of $\alpha_{i}$, the largest signal Constellation for each subcarrier for which $\mathrm{P}_{\mathrm{i}}$ is below $\mathrm{P}_{\mathrm{T}}$ is calculated.

2) The current values of $b$ and the multichannel SNR $\alpha_{m c}$ are calculated.

3) To find the maximum average number of bits $\left(b_{\max }\right)$ per subcarrier that satisfies $\mathrm{P}_{\mathrm{T}}$

$$
\overline{\mathrm{b}}_{\max }=\log _{2}\left\{1-\frac{1.8 \alpha_{\mathrm{mc}}}{\ln \left(6 . \mathrm{P}_{\mathrm{T}}\right)}\right\}
$$

4) The number of extra bits $I=N .\left(\bar{b}_{\max }-\bar{b}\right)$ that can be added to the OFDMA symbol is then found.

\subsection{Proposed Bit Loading Algorithm}

Although many studies on adaptive bit loading [1][3] algorithms make the assumption that the subcarrier SNR values are perfectly known, this is not the case in reality. Therefore, it is necessary to investigate the impact of imperfect subcarrier SNR information on the throughput of these systems.

In particular, two sources of error in subcarrier SNR information are studied:

A) Channel estimation error

B) Quantization error.

\section{A.SNR Channel Estimation Noise Model}

Channel estimation in multicarrier systems, especially WLAN [9] systems, uses predefined training symbols across the subcarriers intermittently to extract channel characteristics. From these characteristics, subcarrier SNR values are computed and used by the adaptive bit loading algorithms.

The errors accompanying the channel estimates also get translated into subcarrier SNR values, resulting in a corrupted

SNR value for subcarrier $\mathrm{n}$. This can be modeled by $\hat{\alpha}_{n}$ $=\alpha_{n}+\mathrm{n}_{\mathrm{e}}$, where $\alpha_{n}$ is the actual SNR value for subcarrier $\mathrm{n}$ and $\mathrm{n}_{\mathrm{e}}$ is the error due to the channel estimation process.

\section{B.SNR Quantization Noise Model}

The adaptive bit loading algorithms require the translation of subcarrier SNR values into Pn values, a look-up table is employed. However, this implies that the subcarrier SNR values must be quantized.

We must determine where to place the quantizer reproduction levels $d_{k}$ in order to minimize the quantization error for all the modulation schemes. Since we want adequate resolution of the BER waterfall curves around $\mathrm{P}_{\mathrm{T}}$, the output levels should be concentrated at that point. Given $q$ bits to represent a quantizer reproduction level, the number of levels is defined as $2 q$. The following algorithm tries to achieve this through a suboptimal placement of $d_{k}$.

1) Determine the pair of SNR values to obtain the probability of bit error values $\mathrm{P}_{\mathrm{n}}$ that are two orders of magnitude above and below $\mathrm{P}_{\mathrm{T}}$ for each modulation scheme, thus forming regions $\mathrm{Q}_{\mathrm{k}}$, for $k=1, \ldots, B$, where $B$ is the number of modulation schemes.

2) For the $B$ modulation schemes, put $2^{\mathrm{q}} / \mathrm{B}$ output levels uniformly in $\mathrm{Q}_{\mathrm{k}}$ for all $k$. In the case of overlapping regions, combine them and their allocation of output levels, distributing the levels uniformly across the combined region. By Distributing $\mathrm{d}_{\mathrm{k}}$, where $k=0, \ldots 2^{\mathrm{q}}-1$, In this way, the 
BER waterfall curves are ensured of quantization with enough resolution.

\section{RESULTS AND DISCUSSIONS}

In this simulation an OFDMA system is presented with $\mathrm{N}=512$ subcarriers and $\mathrm{K}=16$ users. The initial signal constellation is 8-QAM to all users. The resource (subcarriers) for all users is allocated using Ordered Subcarrier selection algorithm [8]. The resulting OFDMA signal is transmitted through a Rayleigh fading channel with 10 taps. The exponential power delay profile is used and each of the channel taps has complex valued gain which in turn has zero mean and variance $\sigma^{2}$.

The adaptive bit loading algorithm is incorporated in all the subcarriers as per the equation and signal constellation initially assigned to all the subcarriers is subject to change depending on the channel estimates at various subcarriers. Essentially, the subcarriers which encounter high values of gain through the channel are used to transmit greater number of bits compared to the subcarriers those have relatively low gain in their power delay profile.

In this simulation two types of Rayleigh channel models are considered. Rayleigh uncorrelated and Rayleigh correlated. Plots are made between BER and $\mathrm{E}_{\mathrm{b}} / \mathrm{N}_{\mathrm{o}}$ for all the simulation results using MATLAB 7.9

\subsection{Simulation Results}

As per M Maniraj, [14] simulation result shows the relationship between Energy per bit $\left(\mathrm{E}_{\mathrm{b}}\right)$ to the spectral noise density (No) and bit error rate (BER). Simulation results are shown for Adaptive Power loading and Bit loading [11] for Rayleigh uncorrelated fading and Rayleigh correlated fading. In the adaptive bit loading algorithm it is essential to have knowledge of channel gain at different frequencies of the channel. However the transmitter has to avail the CSI through pilot carriers transmitted in the signal and has to estimate the CSI based on the feedback information it gets from the receiver.

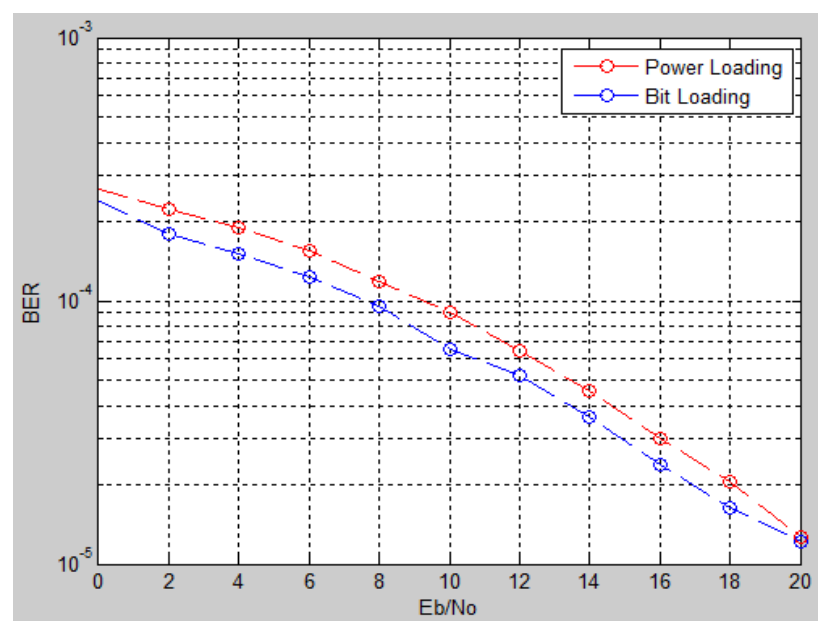

Figure 2. Adaptive Power loading and Bit loading for Rayleigh uncorrelated fading

Figure 2 shows the simulation result of Adaptive Power loading and Bit loading for Rayleigh uncorrelated fading.

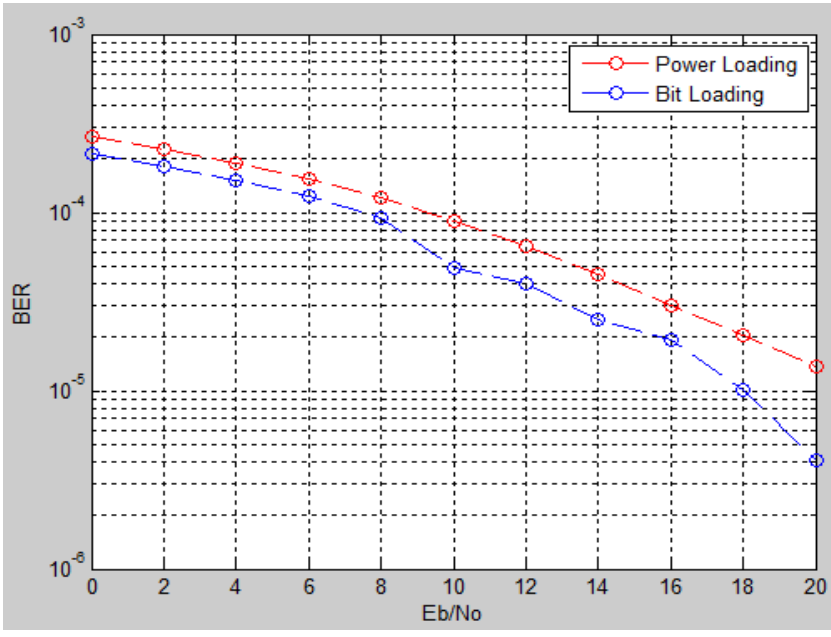

Figure 3. Adaptive Power loading and Bit loading for Rayleigh correlated fading

Figure 3 shows the simulation result of Adaptive Power loading and Bit loading for Rayleigh correlated fading.

\section{CONCLUSION}

Dynamic resource allocation had been implemented in OFDMA systems with fairly in low complexity and with a better performance in terms of the $\mathrm{E}_{\mathrm{B}} / \mathrm{N}_{\mathrm{O}}$ and BER.It is shown in this paper that, combination of adaptive bit loading with OSSA algorithm in resource sharing in OFDMA systems shows significant improvement in the performance of the system over adaptive power loading. Particularly this is suitable where higher frequency range is used and it offers certain kind of flexibility in terms of the scaling of the system and also it is robust to both correlated and uncorrelated fading.

Simulation result of Adaptive Power loading and Bit loading for Rayleigh uncorrelated fading shows that, it has very low BER for the increasing values of $\mathrm{E}_{\mathrm{b}} / \mathrm{N}_{\mathrm{o}}$ which produces low computational complexity and better performance in OFDMA systems

\section{REFERENCES}

[1] Dardari D, "OSSA for OFDMA based high speedWLANs" IEEE Transactions on Wireless Communications, Vol. 3, No 5, pp 1452 - 1458.

[2] Myeon-gyun Cho, Woohyun Seo, and Daesik Hong "AJoint Feedback Reduction Scheme Using Delta Modulation for Dynamic Channel Allocation in OFDMA Systems" IEEE Transactions on wireless Comm., Vol. 6, NO. 1, JANUARY 2007 pp.46-49

[3] Andre Noll Barreto and Simeon Furrer "Adaptive Bit Loading for Wireless OFDM Systems" 2001 IEEE pp. $88-92$

[4] Bohge.M, J. Gross, M. Meyer, and A. Wolisz.Dynamic Resource Allocation in OFDM Systems: An Overviewof Cross-Layer Optimization Principles and techniques IEEE Network, 21(1):53.59, 2007.

[5]

Khaled Ben Letaief, Ying Jun Zhang "Dynamic Multiuser Resource Allocation and adaptation for Wireless Systems" IEEE Wireless Communications, August 2006 pp.38-47 
[6] Campello.J, "Optimal discrete bit loading for multicarrier modulation systems," in Proc. IEEE Symposium on Information Theory 1998, pp.193.

[7] Cheong Yui Wong, Roger S. Cheng "Multiuser OFDMwith adaptive Sub-carrier, bit and Power allocation" IEEE journal on selected areas in communications

[8] Wong et al C.Y., "Multiuser OFDM with Adaptive Subcarrier, Bit and Power Allocation," IEEE JSAC, vol. 17 , no. 10 , Oct. 1999 , pp. $1747-58$.

[9] SangJun Ko , Joo Heo , KyungHi Chang "Aggressive Subchannel allocation algorithm for efficient dynamic Channel allocation in Multi-User OFDMA system" The 17th Annual IEEE International Symposium on Personal, Indoor and Mobile Radio Communications (PIMRC'06)

[10] Liu .Q, S. Zhou, and G. B. Giannakis, "Queuing withAdaptive Modulation and Coding over Wireless Links:Cross-Layer Analysis and Design," IEEE Trans. Wireless Comm, vol. 4, no. 3, May 2005, pp. 1142-53.
[11] Natalia Y. Ermolova and Boris Makarevitch "Low Complexity Adaptive Power and Subcarrier Allocation for OFDMA" IEEE Transactions on wireless comm., Vol. 6, 2007 pp. 433-437

[12] Punal.O, H. Escudero, and J. Gross, "Performancecomparison of loading algorithms for 80 $\mathrm{MHz}$ IEEE 802.11 WLAN's," in Proc. IEEE 73rd Vehicular Tech. Conference (VTC 2011 spring), Budapest, Hungary, May 2011.

[13] M Maniraj "Dynamic Resource Allocation and Rate Adaptation in OFDMA Systems", Project report submitted to Sathyabama University, Chennai, India, 2012

[14] Thoen. S, L. Vander Perre, B. Gyselinckx, M.Engels and $\mathrm{H}$. de Man, "Adaptive loading in the downlink of OFDMA-based wireless local networks", in Proc. VTC 2000, 